WIE INTERNATIONAL

IST DIE LITERATURWISSENSCHAFT? 


\title{
Wie international ist die Literaturwissenschaft?
}

\author{
Methoden- und Theoriediskussion \\ in den Literaturwissenschaften: \\ kulturelle Besonderheiten \\ und interkultureller Austausch \\ am Beispiel \\ des Interpretationsproblems \\ (1950-1990)

\begin{abstract}
Herausgegeben
von Lutz Danneberg und Friedrich Vollhardt in Zusammenarbeit

mit Hartmut Böhme und Jörg Schönert
\end{abstract}


Die Deutsche Bibliothek - CIP-Einheitsaufnahme

Wie international ist die Literaturwissenschaft? : Methoden- und Theoriediskussion in den Literaturwissenschaften: kulturelle Besonderheiten und interkultureller Austausch am Beispiel des Interpretationsproblems ; $(1950$ - 1990) / hrsg. von Lutz Danneberg und Friedrich Vollhardt in Zusammenarbeit mit Hartmut Böhme und Jörg Schönert. Stuttgart ; Weimar : Metzler, 1996

ISBN 978-3-476-01382-8

NE: Danneberg, Lutz [Hrsg.]

ISBN 978-3-476-01382-8

ISBN 978-3-476-03631-5 (eBook)

DOI 10.1007/978-3-476-03631-5

Dieses Werk einschließlich aller seiner Teile ist urheberrechtlich geschützt. Jede Verwertung außerhalb der engen Grenzen des Urheberrechtsgesetzes ist ohne Zustimmung des Verlages unzulässig und strafbar. Das gilt insbesondere für Vervielfältigungen, Übersetzungen, Mikroverfilmungen und die Einspeicherung und Verarbeitung in elektronischen Systemen.

(C) 1996 Springer-Verlag GmbH Deutschland

Ursprünglich erschienen bei J.B. Metzlersche Verlagsbuchhandlung und Carl Ernst Poeschel Verlag GmbH in Stuttgart 1996 


\section{Inhalt}

Vorwort ..................... IX

HARTMUT BöHME (Berlin): Internationalisierung als Aufgabe.

Rede zur Begrüßung der Teilnehmer . . . . . . . . . . . . . . . . 1

LuTZ DANNEBERg (Berlin/Bern) und Jörg Schönert (Hamburg):

Zur Transnationalität und Internationalisierung von Wissenschaft . . . . 7

WILHELM VoSSKAMP (Köln): Jenseits der Nationalphilologien:

Interdisziplinarität in der Literaturwissenschaft . . . . . . . . . . . . 87

Lutz Danneberg (Berlin/Bern): Interpretationstheorie und fremdkulturelles Verstehen . . . . . . . . . . . . . . . . . . . . . . . . 99

KlaUs WeIMAR (Zürich): Text, Interpretation, Methode.

Hermeneutische Klärungen . . . . . . . . . . . . . . . . . . 110

DAVID E. WELLBERY (Baltimore): Interpretation versus Lesen.

Posthermeneutische Konzepte der Texterörterung . . . . . . . . . . . . 123

JürGEN FoHRMANN (Bonn): Annotationen zu David Wellberys

Thesen ... . . . . . . . . . . . . . . . . . . 139

KLaus Weimar (Zürich): Annotationen zu David Wellberys Thesen . . . 142

WolfGang Pross (Bern): Die Verspätung der wissenschaftsgeschichtlichen Debatte in der deutschen Literaturwissenschaft . . . . . . . . 145

KARL ROBERT MANDELKOW (Hamburg): Goethe-Forschung als

Paradigma literaturwissenschaftlicher Methodendiskussion im 20. Jahrhundert . . . . . . . . . . . . . . . . . . 168

Hartmut Böhme (Berlin): Die Wiederaufnahme der hermeneutischen Fragestellung: Anstöße aus den Nachbardisziplinen

ERNSTPETER MAURER (Bonn): Scriptura sacra sui ipsius interpres.

Grundprobleme theologischer Hermeneutik . . . . . . . . . . . . . 186 
ERnST-OTto Gerke (Hamburg): Verstehen heißt Schlüsse ziehen. Kritische Überlegungen zur Behandlung des Verstehensproblems in der pragmatischen Sprachanalyse . . . . . . . . . . . . . . . . 205

LUDWIG JÄGER (Aachen): Die Internationalisierung der Linguistik und der strukturalistische Purismus der Sprache. Ein Plädoyer für eine hermeneutisch-semiologische Erneuerung der Sprachwissenschaft . . .

Friedrich Vollhardt (Magdeburg): Exemplarische Wege des TheorieTransfers in Westeuropa . . . . . . . . . . . . . . . . . . . . . . 283

Chryssoula Kambas (Osnabrück): Theorie-Transfers und Internationalisierung der Literaturgeschichte . . . . . . . . . . . . . 287

WALTER ERHART (Göttingen): Internationalisierung - Pluralisierung Interpretation. Eine wissenschaftsgeschichtliche Fallstudie am Beispiel des Internationalen Referatenorgans Germanistik . . . . . . . 305

REINOLD WERNER (Paris): Verwaltungszwang versus Innovation. Über Berührungsängste der französischen Germanistik am Beispiel der Strukturalismus-Debatte . . . . . . . . . . . . . . . . . . . 329

HORST STEINMETZ (Leiden): Assimilation. Zur Aufnahme der Rezeptionsästhetik in den Niederlanden . . . . . . . . . . . . . . . . . 337

GERD LABROISSE (Amsterdam): Theorie- und Methodendiskussionen in der Forschung zur DDR-Literatur und ihrer rezeptiven Aneignung. Zum Amsterdamer Forschungsprojekt . . . . . . . . . . . . . . . 34

Jörg Schönert (Hamburg): Nationale Konstellationen der TheorieRezeption in Osteuropa

KLAUS STÄDTKE (Bremen): Russische Interpretationstheorien: Konstellationen ihrer internationalen Rezeption . . . . . . . . . . . . . 362

AlEXANDER MichaILOw (Moskau): Interpretieren und Verstehen vor dem Erfahrungshintergrund der russischen Literaturwissenschaft . . . . 374

WOLF SCHMID (Hamburg): Zur Theorie-Rezeption in der russischen und tschechischen Literaturwissenschaft

KÁROLY CsúrI (Szeged): Ein Präzedenzfall für die Internationalisierung der Wissenschaften: Zur semiotischen Literaturtheorie von Zoltán Kanyó

UTZ RIESE (Berlin): Poststrukturalismus/Postmoderne und das Nahen der Wende in der DDR

\section{Friedrich Vollhardt (Magdeburg): Nationale Konstellationen der} Theorie-Rezeption in außereuropäischen Kulturbereichen

Klaus Meyer-Minnemann und Katharina Niemeyer (Hamburg):

Die Rezeption des französischen Strukturalismus in Mexiko . . . . . . 444

REINHARD EMMERICH (Hamburg): Nähe und Ferne. Schwierigkeiten im Umgang mit chinesischer Dichtung . . . . . . . . . . . . . 
Peter PöRTner (München): Aneignung durch Enteignung, ein japanischer Weg. Flankierende Notizen zum Fremdverständnis, zur japanischen Literaturwissenschaft und zum Übersetzungsproblem . . . 478

Hartmut Böhme (Berlin): Neue Perspektiven: Vergleichende Interkulturelle Literaturwissenscht? . . . . . . . . . . . . . . . . . . . 493

LILIANE WeISSBERG (Philadelphia): Zur Ausstellung des Fremden:

Literaturkritik als cultural studies . . . . . . . . . . . . . . . . . . . . 499

Peter V. Zima (Klagenfurt): Komparatistik als Metatheorie. Zur interkulturellen und interdisziplinären Perspektive der Vergleichenden Literaturwissenschaft . . . . . . . . . . . . . . 532

ALOIS WIERLACHER (Bayreuth): Internationalität und Interkulturalität. Der kulturelle Pluralismus als Herausforderung der Literaturwissenschaft. Zur Theorie Interkultureller Germanistik . . . . . . . . . 550

LEO KREUTZER (Hannover): Eigensinn und Geschichte. Überlegungen zu einer Literaturwissenschaft als interkultureller Entwicklungsforschung . . . . . . . . . . . . . . . . 591

NoRBERT NDONG (Yaoundé): Aspekte der Beziehung zwischen afrikanischer und europäischer Literaturwissenschaft . . . . . . . . 600

Namenregister ...................... . . 6 621 


\section{Vorwort}

Wie bedeutsam ist der internationale Austausch für die Geisteswissenschaften? Sind Transnationalität, Internationalität, Interkulturalität und Interdisziplinarität mehr als nur Schlagworte in den bildungs- und wissenschaftspolitischen Debatten? Solche Fragen klingen vielleicht naiv. Werden sie jedoch mit dem Anspruch gestellt, das damit verbundene Problemspektrum systematisch zu beschreiben, dann zeigt sich schnell, daß die unterschiedlichen (historisch oder aktuell orientierten) Forschungsbeiträge nicht im Sinne eines etablierten Forschungsstandes ausgewertet und die Leitfragen theoretisch nicht hinreichend gelöst sind. Die Brisanz der Fragen enthüllt sich zudem erst bei einem zweiten Blick auf das Problemfeld. Die Vorstellung einer universalen, allgemein verbindlichen Methodologie (auch für die textinterpretierenden Wissenschaften) entstammt der westlichen Denktradition. Dieser normative Rationalitätsbegriff erscheint heute obsolet; der intensiver gewordene Austausch mit einer Vielzahl regionaler Kulturen hat das Bewußtsein für die >Inkommensurabilität< verschiedener Wissensformen geschärft. Doch gleichzeitig setzt die moderne Wissenschaft auf beschleunigte, weitreichende Kommunikation und damit auf eine >welteinheitliche<, die Vielfalt der Kulturen verbindende Forschung. Das sind nur einige der Probleme, auf welche die vorliegenden Studien reagieren.

Die Mehrzahl der hier zusammengestellten Beiträge sind für das Zweite Internationale Hamburger Kolloquium "Zu Problemen der Literaturinterpretation und Literaturgeschichtsschreibung " (Hamburg, 13. bis 16. Oktober 1992) verfaßt worden. Alle Vorlagen wurden zuvor an die Teilnehmer verschickt, so daß die Tagung ganz im Zeichen der Diskussion stand. Wichtige Ergebnisse des Gesprächs sind von den Autoren in die Druckfassung der Beiträge eingearbeitet worden; die den Beiträgen vorangestellten Einleitungen der Veranstalter pointieren Ergebnisse des Diskussionsverlaufs und eröffnen weiterführende Perspektiven. Reinold Werner mußte kurzfristig für das Kolloquium absagen, seine Vorlage ist in die ursprünglich vorgesehene Folge der Beiträge einbezogen. Wolf Schmid und Peter V. Zima konnten nachträglich für die Mitarbeit gewonnen werden; für ihre Bereitschaft, sich am vorliegenden Band zu beteiligen, sei ihnen herzlich gedankt. Auch der Beitrag »Zur Transnationalität und Internationalisierung von Wissenschaft $\ll$ ist nach dem Kolloquium entstanden. Die Diskussionen hatten gezeigt, daß es notwendig ist, die vielfach benutzten Termini und Begriffe 
systematisch zu explizieren und gegeneinander abzugrenzen. Dieses Ziel verfolgt die Studie von Lutz Danneberg und Jörg Schönert, in dem die Beispiele für die Argumentation nicht auf die Geisteswissenschaften beschränkt werden.

Die Beiträge stellen dar, welchen Einfluß die internationale Diskussion auf die methodischen und theoretischen Konzepte in den nationalkulturellen Philologien ausübt. Gefragt wird insbesondere nach den Umformungen und Verfestigungen, die diese Konzepte im Prozeß 3 des internationalen Wissenschaftsaustauschs erfahren. Die dadurch gewachsenen Herausforderungen lassen sich an unterschiedlichen Vermittlungsformen ablesen: Offensiv vertretene >Innovationen<, die gewachsene Verständigungsmuster verdrängen wollen, stehen neben Versuchen, vorhandene Konzeptionen zu stabilisieren, oder informierenden Darlegungen, die nur der Diversifikation des methodischen und theoretischen Bestandes dienen. Am Beispiel der textorientierten Interpretation wird geprüft, inwieweit die internationale (und interkulturelle) Kommunikation ein verbindliches hermeneutischen Wissen hervorgebracht oder relativiert hat.

Vorgesehen waren darüber hinaus Darstellungen zu den Aufgaben und Aktivitäten wichtiger wissenschaftsorganisatorischer und wissenschaftspolitischer Institutionen für den internationalen Austausch. Aus unterschiedlichen Gründen ließ sich dieses Vorhaben leider nur in Ansätzen realisieren (vgl. die Beiträge von Wilhelm Voßkamp und Walter Erhart).

Die Herausgeber danken Stefan Andriopoulos und Christian Weller für ihre Arbeit an den Diskussionsprotokollen, Erika Schorno für das Korrekturlesen der Druckvorlage, Wilhelm Schernus für die Gesamtredaktion des Bandes, dem Präsidenten der Universität Hamburg für die Eröffnungsrede beim Kolloquium und die Bewirtung der Teilnehmer am ersten Abend, schließlich dem Metzler-Verlag für die Übernahme des Bandes in sein Programm. Die Deutsche Forschungsgemeinschaft gewährte einen Druckkostenzuschuß und trug zusammen mit der Behörde für Wissenschaft und Forschung der Freien und Hansestadt Hamburg die Finanzierung des Kolloquiums; beiden Institutionen danken wir für die hilfreiche Unterstützung. 